\title{
Variability of Cerebral Deep Venous System in Preterm and Term Neonates Evaluated on MR SWI Venography
}

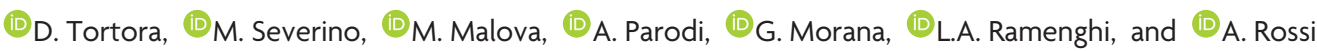

\begin{abstract}
BACKGROUND AND PURPOSE: The anatomy of the deep venous system is characterized by a great variability that might play an important role in the pathogenesis of brain lesions in the preterm brain. The aim of this study was to compare the anatomy of cerebral subependymal veins evaluated on SWI venography in 3 groups of neonates with normal brain MR imaging (very preterm [gestational age $<32$ weeks], moderate-to-late preterm [gestational age $\geq 32$ to $\leq 37$ weeks], and term neonates [gestational age $>37$ weeks]) and to evaluate the influence of preterm birth on development of subependymal veins.
\end{abstract}

MATERIALS AND METHODS: SWI venographies of 84 very preterm, 31 moderate-to-late preterm, and 50 term neonates were retrospectively evaluated. Subependymal vein anatomy was classified into 6 different patterns: type 1 represented the classic pattern and types $2-6$ were considered anatomic variants. A $\chi^{2}$ test was used to evaluate differences between the distributions of subependymal vein patterns.

RESULTS: A significant difference $(P=.011)$ was noticed between the 6 patterns based on gestational age. Type 1 was more frequent in term neonates (68\%) than in both very preterm (41.7\%) and moderate-to-late preterm neonates (56.5\%). Anatomic variants were more common in very preterm neonates (66\%) than in both moderate-to-late preterm (41\%) and term neonates (36\%). Interhemispheric asymmetry was more frequent in very preterm (59.5\%) and moderate-to-late preterm neonates (51.6\%) than in term neonates $(34 \%$; $P=.017)$. Sex and monozygotic twin birth did not significantly affect the frequency of subependymal vein patterns $(P=.0962)$.

CONCLUSIONS: The deep venous system of the neonatal brain shows a large spectrum of anatomic variants with higher variability of subependymal vein anatomy in preterm than term neonates, likely related to the influence of the preterm birth and epigenetic factors on subependymal vein development.

ABBREVIATIONS: $\mathrm{ASV}=$ anterior septal vein; $\mathrm{TSV}=$ thalamostriate vein; $\mathrm{DLV}=$ direct lateral vein; $\mathrm{ICV}=$ internal cerebral vein; $\mathrm{SV}=$ subependymal veins

$\mathrm{T}$ he cerebral deep venous system includes veins that course into the lateral ventricles from the surrounding white matter and basal ganglia and then exit into the transverse fissure, draining into the internal cerebral veins (ICVs). ${ }^{1}$ These veins are characterized by their subependymal location in the lateral ventricles and by their centripetal direction of blood flow. In 1964, Wolf and Huang $^{2}$ classified the deep venous system into 2 main groups: the deep medullary veins and the subependymal veins (SV). The deep medullary veins are small vessels, arranged in wedge-shaped patterns within the white matter of the cerebral hemispheres, directly participating in the drainage of white matter via the SV into the

Received April 12, 2016; accepted after revision May 23.

From the Neuroradiology Unit (D.T., M.S., G.M., A.R.) and Neonatal Intensive Care Unit (M.M., A.P., L.A.R.), Istituto Giannina Gaslini, Genoa, Italy.

Please address correspondence to Domenico Tortora, MD, Neuroradiology Unit, Istituto Giannina Gaslini, Via Gerolamo Gaslini 5, 16147 Genoa, Italy; e-mail: domenicotortora@gaslini.org

http://dx.doi.org/10.3174/ajnr.A4877
ICVs. The SV bilaterally surround the medial and lateral edges of lateral ventricles and are classified based on the portion of the lateral ventricles in which they lie (Fig 1).

The first anatomic studies described the cerebral deep venous system based on ex vivo angiographic planes. ${ }^{3}$ Today, MR imaging can be used as an alternative, noninvasive method to characterize the anatomy of cerebral arteries and veins. Several types of angiographic sequences have been developed to unravel the signal of the blood flow in the arteries (arterial MRA) and in the veins (venous MRA). Venous MRA techniques demonstrate the anatomy of larger vessels, such as the cerebral dural veins, but often fail to depict the blood-flow signal in the smaller veins of the deep venous system. ${ }^{4}$ However, a relatively new sequence, SWI, can depict small vessels and venous structures rich in deoxygenated blood. ${ }^{5}$ Indeed, this 3D, fully velocity-compensated gradient recalled-echo sequence uses deoxyhemoglobin as an intrinsic contrast agent, therefore affording in vivo assessment of even smaller cerebral veins. ${ }^{6}$ 


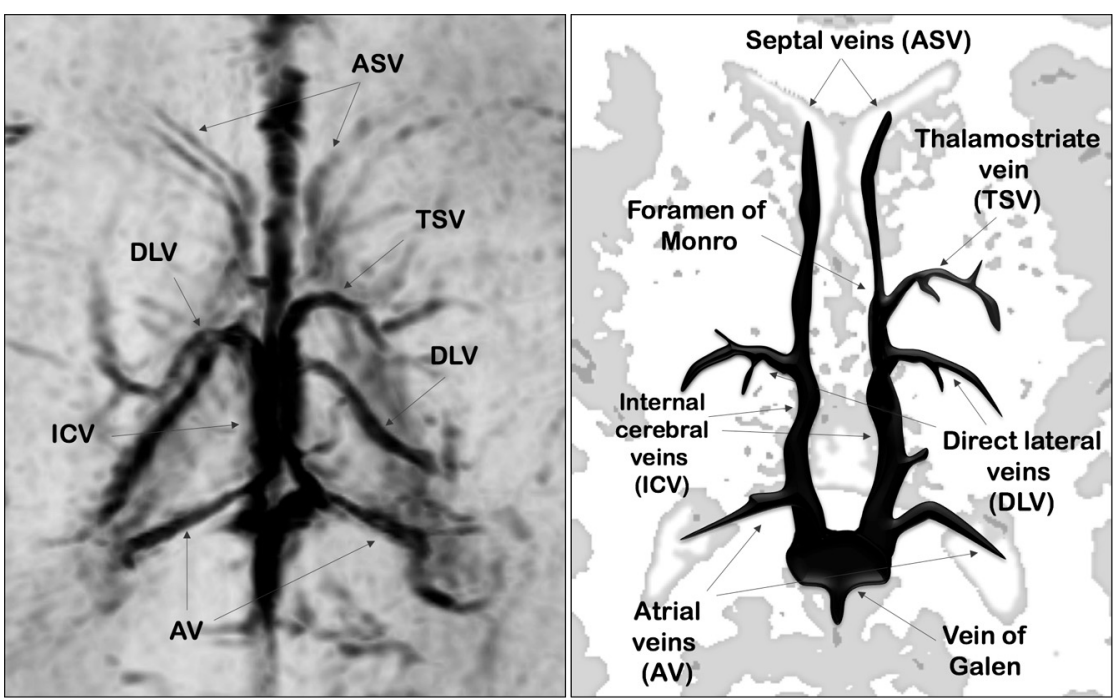

FIG 1. Axial-reformatted SWI venography and corresponding schematic representation of SV.

Table 1: MRI sequence parameters

\begin{tabular}{lcccccccc}
\hline \multicolumn{1}{c}{$\begin{array}{c}\text { Section } \\
\text { Thickness } \\
(\mathbf{m m})\end{array}$} & Matrix & $\begin{array}{c}\text { Intersection } \\
(\mathbf{m m})\end{array}$ & $\begin{array}{c}\text { TR } \\
(\mathbf{m s e c})\end{array}$ & $\begin{array}{c}\text { TE } \\
(\mathbf{m s e c})\end{array}$ & $\begin{array}{c}\text { FA } \\
(\mathbf{d e g})\end{array}$ & $\begin{array}{c}\text { SAR } \\
\text { (W/kg) }\end{array}$ & Bandwidth \\
\hline Axial T1-SE & 3 & $232 \times 110$ & 1 & 780 & 16 & NA & $<2.6$ & 8.2 \\
Axial T2-TSE & 3 & $308 \times 171$ & 1 & 6923 & 140 & NA & 3.1 & 5.6 \\
Coronal T2-TSE & 3 & $232 \times 171$ & 1 & 6782 & 140 & NA & $<3$ & 6.7 \\
3D T1 TFE & 1 & $200 \times 150$ & NA & 9.8 & 4.6 & 10 & $<0.2$ & 2.3 \\
Axial DWI & 4 & $108 \times 104$ & 0.4 & 2530 & 74 & NA & 0.3 & 12.9 \\
\hline
\end{tabular}

Note:-FA indicates flip angle; NA, not available; SAR, specific absorption rate; SE, spin echo; TFE, turbo-field echo. brain lesions detected at early cranial sonography or to evaluate brain involvement after perinatal asphyxia.

Four exclusion criteria were used in this study: presence of brain lesions at MR imaging, history of perinatal asphyxia or any other clinical adverse event, absence of SWI venography in the study protocol, and poor quality of MR images.

Preterm neonates were then classified based on gestational age as very preterm ( $<32$ weeks $)$ and moderate-to-late preterm ( $\geq 32$ to $\leq 37$ weeks). ${ }^{14}$ Among all groups of neonates, the pairs of monozygotic twins were selected, and their SV patterns were compared to identify possible differences in SV anatomy among neonates with identical genes.

\section{Imaging}

MR imaging was performed with a $1.5 \mathrm{~T}$ whole-body system (Achieva 1.5T X-Series; Philips Healthcare, Best, the Netherlands) using an 8-channel dedicated neonatal head array coil. The parents of the neonates provided written informed consent before acquisitions.

All patients were fed before MR im-

The anatomy of the deep venous system is characterized by a great variability ${ }^{4,7}$ that has been ascribed to rearrangement of the main venous drainage of the primitive choroid plexus occurring in the late period of fetal life. ${ }^{3,8}$ This anatomic variability might play an important role in the pathogenesis of brain lesions in the preterm brain. In particular, germinal matrix-intraventricular hemorrhage, the most frequent brain lesion and leading cause of poor neurodevelopmental outcome and motor disability in preterm neonates, ${ }^{9}$ has been associated with acquired and congenital risk factors, ${ }^{10}$ including peculiarities in the venous drainage through the brain deep venous vessels. ${ }^{11-13}$ The aim of this study was to describe the anatomy of SV evaluated on SWI venography in 3 groups of neonates with normal brain MR imaging (very preterm [gestational age $<32$ weeks], moderate-to-late preterm [gestational age $\geq 32$ to $\leq 37$ weeks], and term neonates [gestational age $>37$ weeks]) and to evaluate the influence of preterm birth on SV development.

\section{MATERIALS AND METHODS \\ Patients}

Our institutional review board approved this retrospective study and waived informed consent.

The brain MR imaging studies of 248 consecutive preterm neonates and 83 term neonates acquired from January 2012 to December 2015 were retrospectively evaluated. In preterm neonates, MR imaging was performed at term-equivalent age as a part of a screening program for identification of prematurity-related lesions. Term neonates (gestational age $>37$ weeks) underwent brain MR imaging within 5 days of birth to rule out suspected aging examination to achieve spontaneous sleep and were spontaneously breathing during examination. The need for sedation to prevent head movement was verified with the neuroradiologist based on the infant's state of arousal and the quality of images after the first sequence. Hearing protection was used in all patients. Heart rate and oxygen saturation were noninvasively monitored by pulse oximetry during examination.

All neonates underwent the same MR imaging protocol (Table 1). SWI data were collected with a 3D, fully flow-compensated fast-field echo sequence by using the following parameters: $\mathrm{TE}=48 \mathrm{~ms}, \mathrm{TR}=33 \mathrm{~ms}, 96$ sections, flip angle $=$ $15^{\circ}$, bandwidth $=140 \mathrm{~Hz} / \mathrm{px}$, field of view $=120 \times 120$, acquisition matrix $=512 \times 512$, acquisition voxel size $=0.78 \times$ $0.88 \times 1.5 \mathrm{~mm}$, and reconstructed voxel size $=0.7 \times 0.8 \times 1$ $\mathrm{mm}$. Parallel imaging (sensitivity encoding) with an acceleration factor of 2 resulted in a total acquisition time of 2 minutes and 57 seconds.

\section{Qualitative Analysis of Subependymal Veins}

Phase 1. A pediatric neuroradiologist with 20 years' experience in neonatal neuroimaging first reviewed all the MR imaging studies by using a workstation equipped with a professional DICOM viewer (OsiriX Imaging Software; http://www.osirix-viewer.com) to exclude neonates with brain lesions. Furthermore, image quality of the acquired SWI sequences was assessed by evaluating the general image quality, noise, venous contrast, and presence of motion artifacts. The selected SWI sequences were translated and rotated into the anterior/posterior commissure 


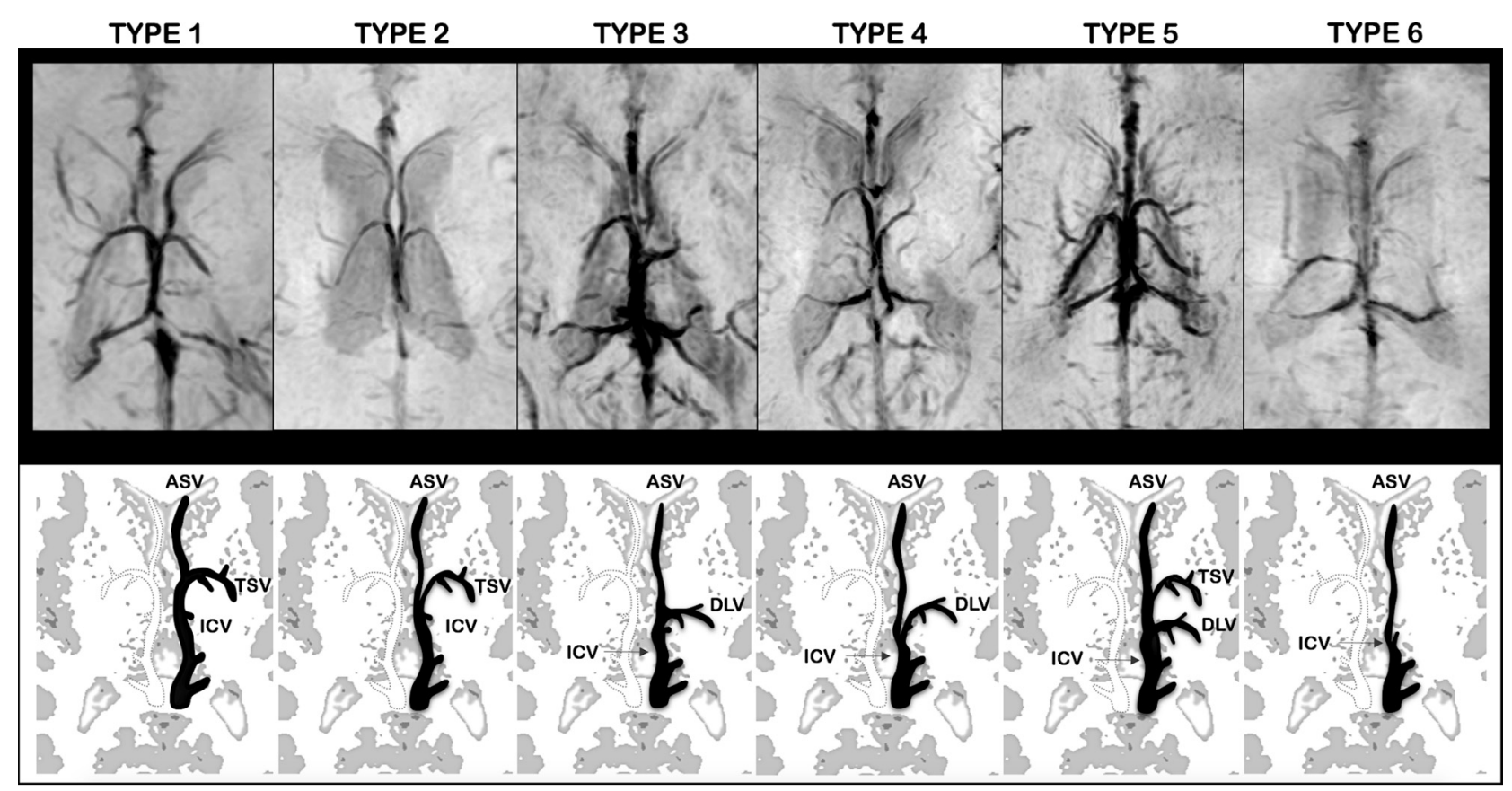

FIG 2. Axial-reformatted SWI venography and corresponding schematic representation of SV patterns. The left side of SWI venography and the black schematic vein represent the anatomic pattern. Type 1: The ASV joined the ICV at the level of the foramen of Monro and the TSV-ICV junction. The DLV was absent. Type 2: The ASV joined the ICV posterior to both the TSV-ICV junction and the foramen of Monro. The DLV was absent. Type 3: The ASV joined the ICV close to the site of DLV-ICV junction, posterior to the foramen of Monro. The TSV was absent. Type 4: The ASV joined the ICV posterior to both the foramen of Monro and DLV-ICV junction. The TSV was absent. Type 5: Both the TSV and DLV were present. Type 6: Both the TSV and DLV were absent. Atrial veins were not included in schematic representations.

Talairach baseline ${ }^{15}$ to standardize the spatial orientation of each brain.

Phase 2. Two neuroradiologists (with 9 and 6 years' experience, respectively), blinded to neonate identity, independently reviewed the SWI sequences selected in phase 1 . They identified the SV on the basis of their anatomic location on axial-reformatted SWI sections. The evaluated SV were the anterior septal vein (ASV), thalamostriate vein (TSV), direct lateral vein (DLV), atrial vein, and ICV (Fig 1).

Subsequently, they evaluated the location of the junction between the ASV and ICV and classified it into 2 categories: 1 ) at the level of the foramen of Monro and 2) posterior to the foramen of Monro.

Phase 3. Based on the results of the phase 2 evaluation, readers independently assigned 1 of 6 patterns of subependymal vein anatomy for each brain hemisphere (Fig 2):

- Type 1: The ASV joined the ICV at the level of the foramen of Monro and the TSV-ICV junction. The DLV was absent. According to Stein and Rosenbaum ${ }^{3}$, this pattern describes the classic anatomy of SV.

- Type 2: The ASV joined the ICV posterior to both the TSV-ICV junction and the foramen of Monro. The DLV was absent.

- Type 3: The ASV joined the ICV close to the site of DLV-ICV junction, posterior to the foramen of Monro. The TSV was absent.

- Type 4: The ASV joined the ICV posterior to both the foramen of Monro and DLV-ICV junction. The TSV was absent.

- Type 5: Both the TSV and DLV were present.

- Type 6: Both the TSV and DLV were absent.
In all SV patterns, the atrial vein was present and had the same course below the splenium of the corpus callosum, joining the posterior end of the ICV.

Furthermore, the SV pattern of each hemisphere was compared with the contralateral to classify each neonate into a symmetric or asymmetric venous pattern group. Finally, the SV anatomic pattern of each pair of monozygotic twins was evaluated to assess if a venous "intertwin" anatomic variability existed.

Phase 4. The same 2 neuroradiologists jointly reviewed the SWI venography of neonates with phase 3 discordant evaluation and were asked to reach a consensus on the SV pattern and interhemispheric symmetry definitions.

\section{Statistical Analysis}

Statistical analysis was performed using SPSS Statistics for Mac, Version 21.0 (IBM, Armonk, New York). The level of significance was set at $P<.05$.

The frequencies and percentages of different SV patterns were calculated for all neonates and separately for the 3 different gestational age groups and for each pair of monozygotic twins. Significant gestational age and sex differences were assessed by a $\chi^{2}$ test. The same test was also used to evaluate significant differences in the distribution of the symmetric and asymmetric SV pattern among the 3 gestational age groups.

\section{RESULTS}

\section{Patients}

The MR imaging studies of 166 patients (133 of 248 [54\%] preterm neonates and 33 of 83 [40\%] term neonates) were excluded from this study based on the exclusion criteria. In particular, 56 
Table 2: Overall frequencies and percentages of SV patterns for each brain hemisphere in the 3 groups of neonates ${ }^{a}$

\begin{tabular}{|c|c|c|c|c|c|c|c|}
\hline \multirow[b]{2}{*}{ Pattern } & \multicolumn{2}{|c|}{ VP } & \multicolumn{2}{|c|}{ MLP } & \multicolumn{2}{|c|}{ TN } & \multirow{2}{*}{$\begin{array}{c}\text { Total } \\
\text { Hemispheres }\end{array}$} \\
\hline & Left & Right & Left & Right & Left & Right & \\
\hline Type 1 & $28(33.3 \%)$ & $42(50 \%)$ & 15 (48.4\%) & 20 (64.5\%) & $36(72 \%)$ & $32(64 \%)$ & 173 \\
\hline Type 2 & $4(4.8 \%)$ & $6(7 \%)$ & 4 (12.9\%) & $0(0 \%)$ & $2(4 \%)$ & $3(6 \%)$ & 19 \\
\hline Type 3 & $36(42.9 \%)$ & $25(29.8)$ & $9(29 \%)$ & $7(22.6 \%)$ & $10(20 \%)$ & $13(26 \%)$ & 100 \\
\hline Type 4 & $7(8.2 \%)$ & $4(4.8 \%)$ & $3(9.7 \%)$ & $1(3.2 \%)$ & $2(4 \%)$ & $2(4 \%)$ & 19 \\
\hline Type 5 & $5(6 \%)$ & $2(2.4 \%)$ & $0(0 \%)$ & $2(6.5 \%)$ & $0(0 \%)$ & $0(0 \%)$ & 9 \\
\hline Type 6 & $4(4.8 \%)$ & $5(6 \%)$ & $0(0 \%)$ & $1(3.2 \%)$ & $0(0 \%)$ & $0(0 \%)$ & 10 \\
\hline
\end{tabular}

Note:-MLP indicates moderate-to-late preterm neonates; TN, term neonates; VP, very preterm neonates. 84 VP neonates; 31 MLP neonates; 50 TN neonates.

Table 3: Frequencies and percentages of interhemispheric symmetry and asymmetry patterns in the 3 groups of neonates

\begin{tabular}{ccccc}
\hline & VP & MLP & TN & $\begin{array}{c}\text { Total } \\
\text { Neonates }\end{array}$ \\
\hline $\begin{array}{c}\text { Pattern } \\
\text { Interhemispheric } \\
\text { symmetry } \\
\begin{array}{c}\text { Interhemispheric } \\
\text { asymmetry }\end{array}\end{array}$ & $34(40.5 \%)$ & $15(48.4 \%)$ & $33(66 \%)$ & 82 \\
Total neonates & 84 & 31 & 50 & 163 \\
\hline
\end{tabular}

Note:-MLP indicates moderate-to-late preterm neonates; TN, term neonates; VP, very preterm neonates.

neonates presented brain lesions and a pathologic clinical history, $37 \mathrm{MR}$ images were affected by motion artifacts, and the remaining 73 neonates lacked SWI venography.

Thus, MR imaging studies of 115 of 248 (46\%) preterm neonates and 50 of $83(60 \%)$ term neonates (13 females; average gestational age, 39 weeks; range 37-41 weeks) were included in this study. Eighty-four of 115 (73\%) preterm neonates were very preterm (40 females; average gestational age, 28 weeks; range, 23-32 weeks), and 31 of 115 (27\%) were moderate-to-late preterm neonates (19 females; average gestational age, 34 weeks; range, 32-36 weeks). Six pairs of female monozygotic twins were identified among the selected neonates; 1 pair was very preterm (gestational age, 26 weeks) and 5 pairs were moderateto-late preterm (average gestational age, 34 weeks; range 32-36 weeks).

\section{Qualitative Analysis of SV}

The consensus reading was necessary in 11 of 165 (6.6\%) neonatal hemispheres (3 very preterm, 4 moderate-to-late preterm, and 4 term). In all discordant cases, the consensus reading was necessary to define the site of ASV-ICV junction to distinguish type 3 and type 4 patterns ( 9 of 11 hemispheres were finally assigned to the type 3 pattern).

No gestational-age difference was observed between the group of males $(n=91)$ and females $(n=74 ; P=.653)$. Moreover, no significantly different frequencies of SV patterns were observed between male and female neonates $(P=.962)$.

The frequencies and percentages of each SV anatomic pattern for the 3 groups of neonates are reported in Table 2. A significant difference was noticed between the 6 anatomic patterns according to gestational age at birth $\left(\chi^{2}=22.977 ; P=.011\right)$. The classic anatomic pattern (type 1) was more frequent in term neonates $(68 \%)$ than in both very preterm $(41.7 \%)$ and moderate-to-late preterm neonates $(56.5 \%)$. The type 3 pattern was the most fre- quent anatomic variant and was more frequent in very preterm $(36.3 \%)$ and moderate-to-late preterm $(25.8 \%)$ neonates. The type 5 and type 6 anatomic patterns were the least frequent and were exclusively observed in very preterm and moderate-to-late preterm neonates.

Table 3 provides frequencies and percentages of interhemispheric asymmetry of SV pattern for the 3 groups of neonates. A significant age difference was observed between the symmetric and asymmetric pattern $\left(\chi^{2}\right.$ $=8.194 ; P=.017)$. The asymmetric pattern was observed more frequently in very preterm $(59.5 \%)$ and moderate-to-late preterm neonates $(51.6 \%)$ than in term neonates $(34 \%)$. Furthermore, the right hemisphere of very preterm and moderate-to-late preterm neonates more frequently presented the classic SV pattern (type 1) than the left hemisphere. Accordingly, the left hemisphere commonly showed variations from the classic patterns.

The analysis of the SV pattern performed on the 6 pairs of monozygotic twins identified different SV patterns between siblings in 5 of 6 pairs $(83.3 \%)$.

\section{DISCUSSION}

The anatomy of the cerebral deep venous system is highly variable in humans. In adults, a systematic observation of the cerebral venous system on MR images has revealed a great anatomic variability of the deep venous system. ${ }^{4,7}$ Four types of deep venous anatomic variants, according to the locations of the ASV-ICV and TSV-ICV junctions in adult brains (types 1-4), have been described to date. ${ }^{4,16}$ The present study with SWI venography demonstrates that the deep venous system of the neonatal brain shows an even larger spectrum of anatomic variants. In particular, the present classification included 2 additional patterns characterized by the simultaneous presence or absence of both TSV and DLV (types 5 and type 6, respectively). Furthermore, in this classification, the posterior location of the ASV-ICV junction relative to the foramen of Monro (types 3 and 4) was not related to an abnormal course of the TSV, but it was considered an anatomic marker of a prominent DLV.

The presence of so many possible anatomic variants likely reflects the complexity of the development of the deep venous system. Indeed, the evolution of cranial veins falls into an orderly sequence related to the changing environment of the fetal brain. The venous drainage in early stages of brain development occurs through the superficial pial venous network in a centrifugal direction. The appearance of the deep venous system is concomitant with the expansion of the cerebral hemispheres and seems to be a necessary circulatory adjustment to the thickening of the periventricular white matter, which encourages an additional centripetal blood flow. ${ }^{1}$ SV are thought to be derivatives of the primitive choroid veins (superior and inferior) that are tributaries of the ICV. As the hemispheres expand, complex mechanisms of integration between the venous structures of the primitive choroid plexus occur, determining the relatively high frequency of variation of SV in the normal neonatal brain. ${ }^{17}$ 
To our knowledge, this is the first study using SWI venography to identify and characterize the venous phenotype associated with preterm birth. The analysis of the SV anatomy of preterm and term neonates revealed significant differences. As expected, the most frequent pattern of SV anatomy matched its classic definition (type 1) in all groups of neonates (ie, the SV were symmetric in both hemispheres, and the TSV was present bilaterally with the ASV-ICV junction located near the foramen of Monro). Conversely, we demonstrated that preterm neonates more frequently presented variations from this pattern. In particular, in very preterm neonates, the DLV replaced a hypoplastic TSV (type 3) with higher frequency than in both moderate-to-late preterm and term neonates (36.3\%, 25.8\%, and 23\%, respectively).

The increased variability of the SV pattern in association with preterm delivery might be explained by the modifications of the environment and angiogenetic factors affecting vascular development after birth. In fact, cerebral vessel development is highly influenced by oxygen levels and fatty acid concentrations. ${ }^{18-22}$ Preterm neonates are delivered into a relatively hyperoxic environment compared with the oxygen levels in the uterus, where relative hypoxia stimulates vessel growth and development. In addition, preterm neonates are deficient in numerous factors normally passed over the placenta during the third trimester of pregnancy, including the essential fatty acids, which are structural and functional constituents of cell membranes and play a fundamental role in vascular development and function. ${ }^{23}$

Intriguingly, in the present study, the proportions of anatomic variants of SV were equally spread among male and female neonates. Sex differences in brain anatomy are known to be present already at birth. In particular, males have greater intracranial and cortical gray-matter volumes than females, and they present larger cranial circumference. ${ }^{24}$ The absence of sex differences in the anatomic pattern of SV in the present study further suggests that brain venous development is not only genetically determined, but also might be influenced by perinatal environmental factors. This hypothesis is also supported by the presence of different SV patterns in almost all pairs of monozygotic twins (83.3\%). Similarly, previous MR studies on monozygotic twins showed high variability in the cortical pattern, thus underlying the strong influence of epigenetic factors on CNS development. ${ }^{25,26}$

We also found a high age-dependent interhemispheric variability of the SV patterns, with very preterm and moderate-to-late preterm neonates presenting an asymmetric SV pattern with higher frequency than term neonates. Hemispheric anatomic asymmetries were first observed in postmortem studies on the fetal and premature cortex, showing that some areas of the right hemisphere mature quicker than the corresponding areas in the left hemisphere. ${ }^{27,28}$ More recent MR imaging studies demonstrated that left-right hemispheric asymmetries in infants are region-specific. For instance, the superior temporal sulcus develops earlier in the right hemisphere, ${ }^{29}$ and the Broca area develops earlier in the left hemisphere. ${ }^{30}$ Recently, Lin et al ${ }^{31}$ demonstrated hemispheric asymmetries and regional differences in cerebral oxygen metabolism, blood flow, and blood volume with near-infrared spectroscopy in premature and term neonates. In particular, they found a higher metabolism and perfusion in the right hemisphere, especially in preterm neonates, supporting current theo- ries about the delay in maturation of the left hemisphere at birth. ${ }^{32,33}$ Interestingly, in the present study, preterm neonates presented the classic SV pattern (type 1) with higher frequency in the right than in the left hemisphere. Considering that the left hemisphere matures later and, thus, is more exposed to the influence of postnatal epigenetic factors, we hypothesize that the variations from the classic SV pattern might represent different forms of a delayed venous development, whereas the type $1 \mathrm{SV}$ pattern might be considered a marker of maturation of the brain structures. Nevertheless, the relationship between development, morphology, and function of the SV itself remains speculative and is worthy of future investigation. In particular, because the degree of prematurity remains a main risk factor for hemorrhagic brain injury, ${ }^{14}$ further studies are needed to explore if the wider variability of SV patterns in very preterm neonates have implications for the development of these lesions.

\section{CONCLUSIONS}

MR SWI venography represents an in vivo, noninvasive method able to describe morphologic, positional, and numeric variants of SV in neonates. In this study, we provided the frequencies of 6 different anatomic patterns in very preterm, moderate-to-late preterm, and term neonates with normal brain MR imaging and demonstrated a significant age difference in SV pattern distribution, likely related to the influence of preterm birth and epigenetic factors on deep venous system development.

\section{REFERENCES}

1. Stephens RB, Stilwell DL. Arteries and Veins of the Human Brain. Springfield, Illinois: C.C. Thomas; 1969

2. Huang YP, Wolf BS. Veins of the white matter of the cerebral hemispheres (the medullary veins). Am J Roentgenol Radium Ther Nucl Med 1964;92:739-55 Medline

3. Stein RL, Rosenbaum AE. Deep supratentorial veins. Section I. Normal deep cerebral venous system. In: Newton TH, Potts DG, eds. Radiology of the Skull and Brain, vol 2. (angiography), book 3 (veins). St. Louis: Mosby; 1974:1903-98

4. Cimşit NC, Türe U, Ekinci G, et al. Venous variations in the region of the third ventricle: the role of MR venography. Neuroradiology 2003; 45:900-04 CrossRef Medline

5. Haacke EM, Xu Y, Cheng YC, et al. Susceptibility weighted imaging (SWI). Magn Reson Med 2004;52:612-18 CrossRef Medline

6. Parodi A, Morana G, Severino MS, et al. Low-grade intraventricular hemorrhage: is ultrasound good enough? J Matern Fetal Neonatal Med 2015;28(suppl 1):2261-64 CrossRef Medline

7. Fujii S, Kanasaki Y, Matsusue E, et al. Demonstration of cerebral venous variations in the region of the third ventricle on phasesensitive imaging. AJNR Am J Neuroradiol 2010;31:55-59 CrossRef Medline

8. Mokrohisky JF, Paul RE, Lin PM, et al. The diagnostic importance of normal variants in deep cerebral phlebography, with special emphasis on the true and false venous angles of the brain and evaluation of venous angle measurements. Radiology 1956;67:34-47 CrossRef Medline

9. Marlow N, Wolke D, Bracewell MA, et al. Neurologic and developmental disability at six years of age after extremely preterm birth. N Engl J Med 2005;352:9-19 CrossRef Medline

10. Ramenghi LA, Fumagalli M, Groppo M, et al. Germinal matrix hemorrhage: intraventricular hemorrhage in very-low-birthweight infants infants: the independent role of inherited thrombophilia. Stroke 2011;42:1889-93 CrossRef Medline

11. Anstrom JA, Brown WR, Moody DM, et al. Subependymal veins in 
premature neonates: implications for hemorrhage. Pediatr Neurol 2004;30:46-53 CrossRef Medline

12. Ramenghi LA, Gill BJ, Tanner SF, et al. Cerebral venous thrombosis, intraventricular haemorrhage and white matter lesions in a preterm newborn with factor $\mathrm{V}$ (Leiden) mutation. Neuropediatrics 2002;33:97-99 CrossRef Medline

13. Volpe JJ. Intracranial hemorrhage: germinal matrix-intraventricular hemorrhage of the premature infant. In: Volpe JJ, ed. Neurology of the Newborn. 5th ed. Philadelphia: Saunders Elsevier; 2008:517-88

14. Sannia A, Natalizia AR, Parodi A, et al. Different gestational ages and changing vulnerability of the premature brain. J Matern Fetal Neonatal Med 2015;28(suppl 1):2268-72 CrossRef

15. Talairach J, Tournoux P. Co-Planar Stereotaxic Atlas of the Human Brain. New York: Thieme; 1988

16. Türe U, Yaşargil MG, Al-Mefty O. The transcallosal-transforaminal approach to the third ventricle with regard to the venous variations in this region. J Neurosurg 1997;87:706-15 CrossRef Medline

17. Padget DH. The cranial venous system in man in reference to development, adult configuration, and relation to the arteries. Am J Anat 1956;98:307-55 CrossRef Medline

18. Malamateniou C, Counsell SJ, Allsop JM, et al. The effect of preterm birth on neonatal cerebral vasculature studied with magnetic resonance angiography at 3 Tesla. Neuroimage 2006;32:1050-59 CrossRef Medline

19. Risau W. Mechanisms of angiogenesis. Nature 1997;386:671-74 CrossRef Medline

20. Plate KH. Mechanisms of angiogenesis in the brain. J Neuropathol Exp Neurol 1999;58:313-20 CrossRef Medline

21. Breier G. Angiogenesis in embryonic development-a review. Placenta 2000;21(suppl A):S11-15 Medline

22. Ferrara N, Gerber HP, LeCouter J. The biology of VEGF and its receptors. Nat Med 2003;9:669-76 CrossRef Medline

23. Crawford MA, Costeloe K, Ghebremeskel K, et al. Are deficits of arachidonic and docosahexaenoic acids responsible for the neural and vascular complications of preterm babies? Am J Clin Nutr 1997; 66(suppl):1032S-1041S Medline

24. Gilmore JH, Lin W, Prastawa MW, et al. Regional gray matter growth, sexual dimorphism, and cerebral asymmetry in the neonatal brain. J Neurosci 2007;27:1255-60 CrossRef Medline

25. Bartley AJ, Jones DW, Weinberger DR. Genetic variability of human brain size and cortical gyral patterns. Brain 1997;120(Pt 2):257-69 CrossRef Medline

26. Steinmetz H, Hergoz A, Huang Y, et al. Discordant brain-surface anatomy in monozygotic twins. $N$ Engl J Med 1994;331:951-52 Medline

27. Chi JG, Dooling EC, Gilles FH. Left-right asymmetries of the temporal speech areas of the human fetus. Arch Neurol 1977;34:346-48 CrossRef Medline

28. Simonds RJ, Scheibel AB. The postnatal development of the motor speech area: a preliminary study. Brain Lang 1989;37:42-58 CrossRef Medline

29. Dubois J, Benders M, Cachia A, et al. Mapping the early cortical folding process in the preterm newborn brain. Cereb Cortex 2008; 18:1444-54 CrossRef Medline

30. Dubois J, Benders M, Lazeyras F, et al. Structural asymmetries of perisylvian regions in the preterm newborn. Neuroimage 2010;52: 32-42 CrossRef Medline

31. Lin PY, Roche-Labarbe N, Dehaes M, et al. Regional and hemispheric asymmetries of cerebral hemodynamic and oxygen metabolism in newborns. Cerebral Cortex 2013;23:339-48 CrossRef Medline

32. Sun T, Collura RV, Ruvolo M, et al. Genomic and evolutionary analyses of asymmetrically expressed genes in human fetal left and right cerebral cortex. Cereb Cortex 2006;16(suppl 1):i18-25 Medline

33. Sun T, Patoine C, Abu-Khalil A, et al. Early asymmetry of gene transcription in embryonic human left and right cerebral cortex. Science 2005;308:1794-98 CrossRef Medline 